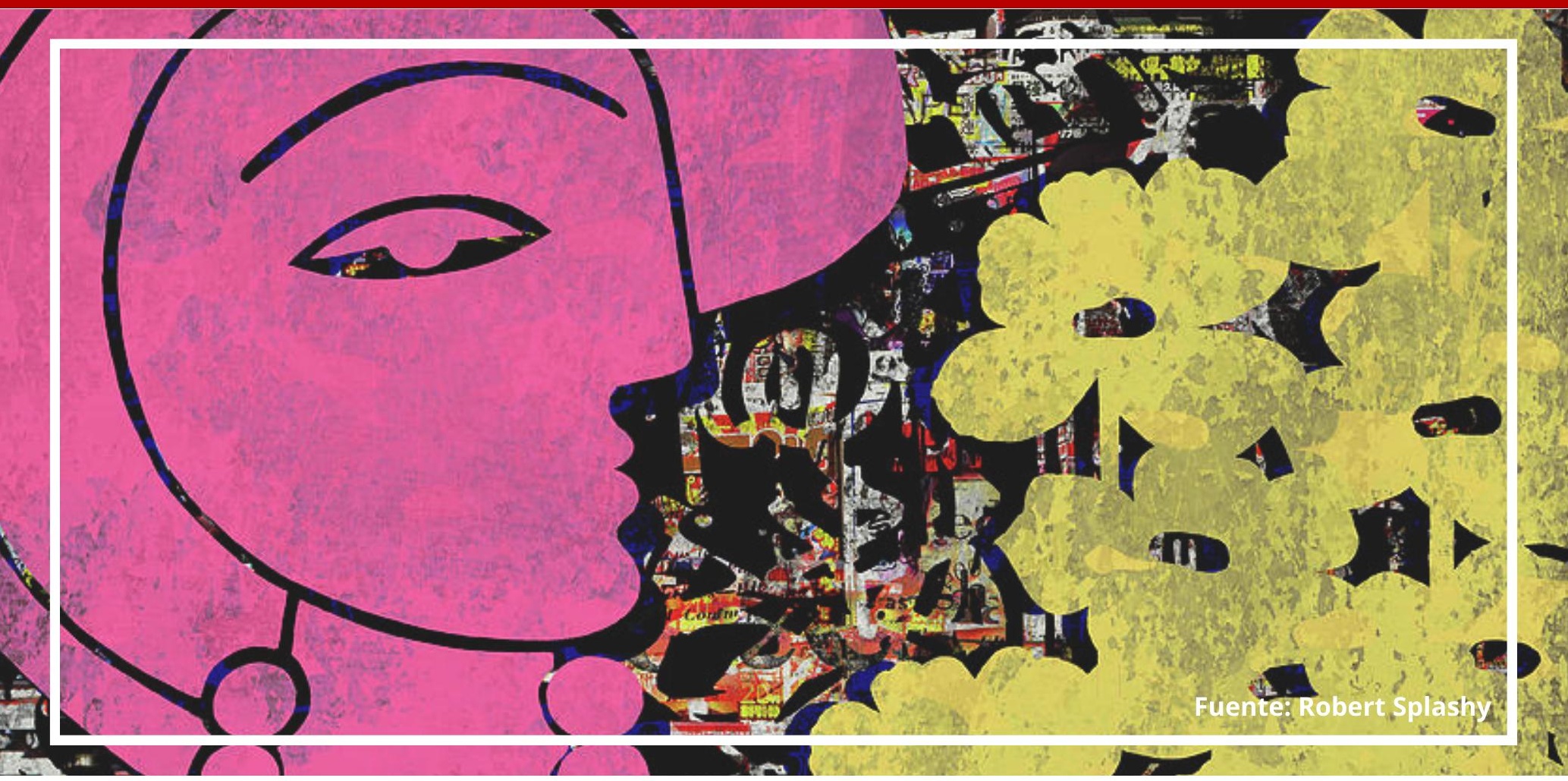

\title{
¿Tiene validez científica el uso de la terapia floral de BACH para la salud?
}

doi: $10.52749 /$ rh.v1i2.5

VÍCTOR ROMÁN LAZARTE iD https://orcid.org/0000-0001-9664-5169

Universidad Nacional Daniel Alcides Carrión: Pasco, Pasco, Perú.

$\checkmark$ victor.md.1998@gmail.com

Cómo citar este artículo:

Román Lazarte, V. (2021). ¿Tiene validez científica el uso de la terapia floral de BACH para la salud? Revista Humanista, 1(2), 32-36. https://doi.org/10.52749/rh.vli2.5

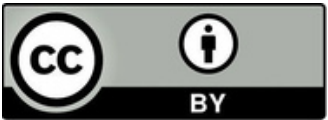




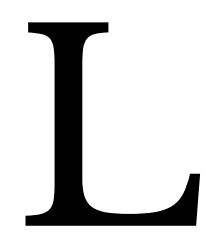

a terapia floral o flores de Bach, se popularizó en la década de los 30' gracias al médico homeópata Edward Bach. Describiéndose como un remedio, asegura tener en cada una de sus 38 esencias un patrón emocional o una personalidad típica; de tal forma esta terapia se usa para curar y prevenir trastornos físicos y/o emocionales mediante el tratamiento de nuestro desequilibrio emocional.

Esta terapia asegura ser muy versátil ya que se puede utilizar con un vasto número de enfermedades, desde crónicas no transmisibles (diabetes, hipertensión, epilepsia, etc.) hasta psiquiátricas (depresión, trastorno por déficit de atención e hiperactividad, etc.) por lo cual es duramente cuestionada en el círculo científico y dentro de la medicina basada en evidencia; aun así muchas casas universitarias, hospitales y profesionales del área (psicólogos y médicos) promueven el conocimiento de estas terapias por ser de naturaleza inocua y no presentar efectos adversos; tal es el caso de EsSalud y universidades como la Universidad Peruana Cayetano Heredia que en Perú ofrecen diplomados y certificación en estas terapias que hasta el momento no tienen un sustento científico valido.

Este artículo tiene por objetivo revelar información científica basada en evidencias sobre la eficacia de la terapia floral mediante una revisión de literatura en diferentes bases bibliográficas (Pubmed, Ebsco, Elsevier, Web of Science) tomando a las revisiones sistemáticas y ensayos controlados aleatorizados como principal fuente de información.

El médico inglés Edward Bach, reconocido a nivel mundial por el descubrimiento $\mathrm{y}$ aplicación de la terapia floral mediante 38 esencias, fue influenciado en gran parte por el médico Hahnemann (también conocido por elaborar terapias homeópatas).

Esta terapia fue diseñada como un sistema de curación emocional y es descrita como medicina homeopática, sus tratamientos supuestamente son seguros y no interfieren con otros medicamentos (Mantle, 1997), por lo cual es bienvenido por las personas que se someten a estos remedios asegurando tener efectos beneficiosos para la salud.

Los remedios que incluyen (para la evaluación objetiva) son: Agrimony: tortura mental detrás de una cara alegre, Aspen: miedo a cosas desconocidas, Haya: intolerancia, Centaury: la incapacidad de decir 'no', Cerato: falta de confianza en las propias decisiones, Cherry Plum: miedo a que la mente ceda, Chestnut Bud: fracaso para aprender de los errores, Achicoria: amor egoísta y posesivo, Clematis: soñando con el futuro sin trabajar en el presente, Manzana de cangrejo: el remedio de limpieza, también por no gustarnos algo de nosotros mismos, Elm: abrumado por la responsabilidad, Genciana: desánimo después de un revés, Gorse: desesperanza y desesperación, Heather: inquietud habladora y egocéntrica, Acebo: odio, envidia y celos, Madreselva: viviendo en el pasado, Hornbeam: cansancio ante la idea de hacer algo, Impatiens: impaciencia, Alerce: falta de confianza, Mimulus: miedo a las cosas conocidas, Mostaza: penumbra profunda sin razón, Roble: el intruso que sigue pasando el punto de agotamiento, Oliva: agotamiento después del esfuerzo mental o físico, Pino: culpa, Castaño rojo: preocupación excesiva por el bienestar de los seres queridos, Rock Rose: terror y susto, Agua de roca: abnegación, rigidez y represión, Scleranthus: incapacidad para elegir entre alternativas, Estrella de Belén: shock, Sweet Chestnut: angustia mental extrema, cuando todo ha sido probado y no queda luz, Vervain: exceso de entusiasmo, Vine: dominación e inflexibilidad, Nuez: protección contra el cambio y las influencias no deseadas, Violeta de agua: autosuficiencia silenciosa que conduce al aislamiento, Castaño Blanco: pensamientos no deseados y argumentos mentales, Avena salvaje: incertidumbre sobre la dirección de uno en la vida, Wild Rose: deriva, resignación, apatía, Willow: autocompasión y resentimiento («The 38 Remedies Quick Reference Guide», s. f.).

Para evaluar la validez científica del tratamiento con flores de Bach se requiere conocer la pirámide de la evidencia en medicina y ciencias de la salud, donde el máximo nivel de evidencia científica es la revisión sistemática y los meta-ánalisis (los cuales también podrían presentar sesgos y requerirían de una lectura crítica como en cualquier otro artículo publicado) seguido de los ensayos clínicos aleatorizados con ciego, doble ciego o incluso triple ciego, en tercer lugar de los estudios cuasiexperimentales, luego los estudios de cohorte y estudios de casos y controles, en 
penúltimo lugar los reportes de casos (de 1 a 10 individuos que conforman el estudio) y al final las opiniones de expertos (Manterola et al., 2014). Con estos conceptos podemos revisar en las fuentes bibliográficas la mejor información.

Los reportes de caso que nos brinda la web de Bach Centre son narraciones cortas, sin rigurosidad científica, no fueron revisados por pares, no fueron publicados en alguna revista indexada y no tienen el esquema de un reporte de caso usual (guía de un reporte de caso disponible en www.care-statement.org).

"El otro día tuve un caso de libro de texto sobre una niña de diez años. Su madre entró en la tienda de salud donde trabajo y practico, harta de la respuesta del médico al dolor de barriga constante de su hija y teníamos una alternativa.

Le pregunté si su hija era bastante sensible y comenzó a decir qué tan nerviosa se pone con lo más mínimo y cómo sufre su trabajo escolar porque no tiene confianza, y que a menudo también tiene dolores de cabeza. También dijo que estaba bastante malhumorada, muy arriba y abajo.

Le pregunté si era indecisa y su madre levantó los ojos hacia arriba y me dijo que habia pasado las últimas semanas decidiendo si iría o no a un viaje escolar. La estaba volviendo loca.

Después de una breve introducción a los remedios florales de Bach, decidió comprar la combinación de emergencia. Tenía ganas de armar una mezcla para ella, pero ella era muy escéptica. Sin embargo, sabía que necesitaba a otros, asi que al final se fue con una combinación de la combinación de emergencia, Larch, Mimulus , Aspen, Scleranthus y Gentian.

Dos días después, su madre regresó con un ramo de flores para mi y me contó cómo su hija habia cambiado de la noche a la mañana. iDespués de dos dosis esa noche, ella había dormido bien y al dia siguiente habia bajado diciendo que no tenía dolor de barriga y que habia tomado una decisión sobre el viaje escolar! Y su maestra había salido al final del día y le preguntó a su madre qué habia pasado, ya que habia sido muy buena en su trabajo escolar ese dia” ("Case study examples», s.f.)
Esta narración nos permite evaluar la calidad científica que posee la terapia floral desde su página web oficial. La revisión en Pubmed y ScienceDirect (repositorios de información en su mayoría revisada por pares) no nos brinda reportes de caso directos, pero sí estudios de cohorte y ensayos clínicos los cuales describiremos en los siguientes párrafos.

En 1998 un artículo de la revista Complementary Therapies in Nursing \& Midwifery explora el uso de la terapia floral en los hospitales en Australia Occidental, área en la que en ese año al menos 16 nosocomios brindaban esa terapia (asegurando el crecimiento de la misma) (Balinski, 1998).

Uno de las primeras revisiones sistemáticas sobre el uso y eficiencia de la terapia floral fue realizado en el 2002, se realizó una búsqueda en 6 bases de datos en donde solo se incluyeron los ensayos clínicos aleatorios de terapias florares en cualquier afección médica, 2 de los 6 ensayos incluidos mostraron resultados positivos pero tuvieron sesgos en la aplicación de las mismas, los 4 ensayos más rigurosos no demostraron efectos superiores al placebo (Ernst, 2002).

En la European Journal of Paediatric Neurology (Q1 en la actualidad) se publicó un ensayo clínico controlado a doble ciego en el año 2005 en donde se evaluaba a la terapia floral como tratamiento para el trastorno por déficit de atención e hiperactividad y su control respectivo con placebo en donde no se encontraron resultados estadísticamente significativos en ningún aspecto a favor de la terapia floral (Pintov et al., 2005). Surgió una duda con la mentalidad de los participantes que se mostraban a favor de las terapias florares, otra variable a considerar era la religiosidad o espiritualidad de cada persona, pero en un estudio transversal (se trabaja con esa variable) tampoco tuvo efecto significativo y aseguran que la terapia funciona como un placebo (Hyland et al., 2006).

En la revista Complementary Therapies in Clinical Practice una revisión de literatura (no confundir con una revisión sistemática) asegura tener un efecto de mejora en los pacientes con dolor provenientes de un cambio emocional positivo, sin un análisis estadístico ni alguna prueba de hipótesis tan solo mostrando frecuencias y comentarios de los pacientes (Howard, 2007).

Otra revisión sistemática publicada en la re- 
vista BMC Complementary and Alternative Medicine, al realizar la selección de los estudios a incluir encuentra un alto riesgo de sesgo, aun así, un punto favorable son los pocos efectos adversos e inocuidad del tratamiento floral, para la ansiedad previo a un examen y en pacientes con trastornos por déficit de atención no hay evidencia de beneficio en comparación con el placebo (Thaler et al., 2009). De misma manera, en la revista Swiss Medical Weekly otra revisión sistemática del 2010 se concluye que los ensayos clínicos más confiables no muestran diferencias entre los remedios florales y los placebos (Ernst, 2010).

A pesar de no tener evidencia científica y de ser una terapia destinada a algún grupo de pacientes en específico, se hizo un ensayo clínico en ratas teniendo como outcome el control de factores de riesgo cardiovascular (controlar el HDL, disminuir la glucosa sanguínea y el colesterol). A pesar de tener resultados "positivos" se usó un análisis multivariado ANOVA, sin tener en cuenta que un análisis con regresión logística, riesgo o probabilidad de asociación serían más confiables (Resende et al., 2014). Otro ejemplo del continuo uso de la terapia floral a pesar de no tener evidencia es el estudio en la revista Journal of Evidence-Based Complementary \& Alternative Medicine, esta vez para el tratamiento del dolor por túnel carpiano en donde solo se ciega a un grupo, pero con un análisis estadístico complejo y con poca población; reduciendo los síntomas de gravedad y proporcionando alivio del dolor (Rivas-Suárez et al., 2017).

En el ámbito nacional desde el año 2000 la OMS reconoce un manual de terapia floral dentro de las terapias alternativas y complementarias (no disponible, pero si está citado en muchos estudios), en el año 2016 se emitió un boletín informativo de medicina complementaria en donde se hace hincapié en un concepto:

"La manera de actuar de estos remedios consiste en elevar nuestras vibraciones $y$ en abrir nuestros canales para que nuestro yo espiritual pueda sentir, en evadir nuestra naturaleza con las virtudes que necesitamos y en subsanar los errores que en nosotros ocasionan daños".

Dr. Edward Bach
Esto acompañado de un efectivo tratamiento del estrés, citando estudios cubanos con poca validez científica teniendo ya para el 2016 las revisiones sistemáticas presentadas en párrafos anteriores (BOLETIN_MEC_JUNIO_2016.pdf, s. f.).

En Perú la distribución de los locales EsSalud que ofrecen el servicio de Medicina complementaria en Perú son en su mayoría los servicios de primer nivel (Luján-Carpio et al., 2014) los cuales generan un gasto sin evidencia al Estado peruano, totalmente diferente de la terapia convencional en donde sí existen estudios con alto nivel de evidencia y actualizándose a cada momento.

Dos claros ejemplos son la diplomatura en terapia floral promovida por la escuela de postgrado de la Facultad de Medicina Humana en la sección de psicología y de otras terapias alternativas sin sustento ni evidencia científica. Así como la Jornada de Medicina Veterinaria Holística de la Dra Nancy Gamboa con diplomatura en esencias florales y certificado del instituto Bach presenta el uso de las esencias florales de Bach con un costo de 200 soles (65 USD).

El último artículo sobre terapias florales en Perú se encuentra en la Revista Peruana de Medicina Integrativa, en donde se hace una revisión de la terapia mencionada en las afecciones orgánicas en donde no se menciona ningún estudio aleatorizado controlado con ciego o doble ciego, y concluyen que puede contribuir una terapéutica de elección en pacientes con hipertensión arterial, vitíligo, alopecia, túnel carpiano (Meza et al., 2019) (algunos que ya se revisaron en párrafos anteriores). Este artículo puede ser replicado con una carta al editor para que hagan una mejor revisión de la literatura publicada y que también hablen más de la poca evidencia científica que tienen hasta el momento.

En este punto la terapia con flores de Bach ya se considera como una pseudociencia ya que escapa de la rigurosidad del método científico y muchas organizaciones presentan evidencia de la misma (Lucero, s. f.), se requieren estudios (con metaanálisis) para descartar todas las malas prácticas clínicas con pseudociencias de por medio, conlleva a un efecto placebo y un gasto innecesario de los recursos del estado peruano. 


\section{Conclusiones}

En efecto, la terapia floral ya entraría en el rubro de pseudociencias ya que se considera "una colección de creencias o prácticas erróneamente consideradas como basadas en un método científico" (González-Méijome, 2017) y que aún sigue en pie, los grandes entes de salud (universidades, hospitales, gremios médicos, etc.) tienen el deber de no confundir a la población y ocasionar un gasto innecesario, además se requieren estudios con la recolección de datos óptima para descartar su uso. La evidencia más rigurosa sugiere que tiene el mismo valor que un placebo.

\section{Referencias}

1.Balinski, A. A. (1998). Use of Western Australian flower essences in the management of pain and stress in the hospital setting. Complementary Therapies in Nursing \& Midwifery, 4(4), 111-117. https://doi.org/10.1016/s1353-6117(98) $80048-6$

2.BOLETIN_MEC_JUNIO_2016.pdf. (s. f.). Recuperado 20 de junio de 2020, de http://www.essalud.gob.pe/downloads/BOLETIN MEC JUNIO 2016. pdf

3.Case study examples. (s. f.). The Bach Centre. Recuperado 20 de junio de 2020, de https://www.bachcentre.com/new/en/remedies/selecting-

remedies/remedy-case-studies/

4.Ernst, E. (2002). «Flower remedies»: A systematic review of the clinical evidence. Wiener Klinische Wochenschrift, 114(23-24), 963966.

5.Ernst, E. (2010). Bach flower remedies: A systematic review of randomised clinical trials. Swiss Medical Weekly, 140, w13079. https://doi.org/10.4414/smw.2010.13079

6.González-Méijome, J. M. (2017). Science, pseudoscience, evidence-based practice and post truth. Journal of Optometry, 10(4), 203-204. https://doi.org/10.1016/j.optom.2017.08.001

7. Howard, J. (2007). Do Bach flower remedies have a role to play in pain control? A critical analysis investigating therapeutic value beyond the placebo effect, and the potential of Bach flower remedies as a psychological method of pain relief. Complementary Therapies in Clinical Practice, 13(3), 174-183. https://doi.org/10.1016/j.ctcp.2007.03.001

8.Hyland, M. E., Geraghty, A. W. A., Joy, O. E. T., \& Turner, S. I. (2006). Spirituality predicts outcome independently of expectancy following flower essence self-treatment. Journal of Psychosomatic Research, $60(1)$

https://doi.org/10.1016/j.jpsychores.2005.06.073

9.Lucero, B. (s. f.). Terapias pseudocientíficas ofrecidas por EsSalud | Científicos.pe, Cientificos Peruanos. Recuperado 20 de junio de 2020, de http://www.cientificos.pe/index.php/2017/07/17/terapiaspseudocientificas-ofertadas-por-essalud/

10.Luján-Carpio, E., Lizarraga-Castañeda, Z., Mayor-Vega, A., Medrano-Canchari, K., Medina-Salazar, H., \& Goicochea-Lugo, S. (2014). El servicio de Medicina complementaria de EsSalud, una alternativa en el sistema de salud peruano. Revista Medica Herediana, 25(2), 105-106.

11.Manterola, C., Asenjo-Lobos, C., \& Otzen, T. (2014). Jerarquización de la evidencia: Niveles de evidencia y grados de recomendación de uso actual. Revista chilena de infectología, 31(6), 705-718. https://doi.org/10.4067/S0716-10182014000600011
12. Mantle, F. (1997). Bach flower remedies. Complementary Therapies in Nursing \& Midwifery, 3(5), 142-144. https://doi.org/10.1016/s1353-6117( $\underline{97}) \underline{80015-7}$

13.Meza, G. S., Bardales, K. M., Cuenca, K. Q., \& Trocones, I. S. (2019). La terapia floral en enfermedades orgánicas. Revista Peruana de Medicina Integrativa, 4(2), 64-68. https://doi.org/10.26722/rpmi.2019.42.122

14. Pintov, S., Hochman, M., Livne, A., Heyman, E., \& Lahat, E. (2005). Bach flower remedies used for attention deficit hyperactivity disorder in children-A prospective double blind controlled study. European Journal of Paediatric Neurology: EJPN: Official Journal of the European Paediatric Neurology Society, 9(6), 395-398. https://doi.org/10.1016/j.ejpn.2005.08.001

15.Resende, M. M. de C., Costa, F. E. de C., Gardona, R. G. B., Araújo, R. G., Mundim, F. G. L., \& Costa, M. J. de C. (2014). Preventive use of Bach flower Rescue Remedy in the control of risk factors for cardiovascular disease in rats. Complementary Therapies in Medicine, 22(4), 719-723. https://doi.org/10.1016/j.ctim.2014.06.008

16. Rivas-Suárez, S. R., Águila-Vázquez, J., Suárez-Rodríguez, B., Vázquez-León, L., Casanova-Giral, M., Morales-Morales, R., \& Rodríguez-Martín, B. C. (2017). Exploring the Effectiveness of External Use of Bach Flower Remedies on Carpal Tunnel Syndrome: A Pilot Study. Journal of Evidence-Based Complementary \& $\begin{array}{lll}\text { Alternative } \quad \text { Medicine, 22(1), } & \text { 18-24. }\end{array}$ https://doi.org/10.1177/2156587215610705

17.Thaler, K., Kaminski, A., Chapman, A., Langley, T., \& Gartlehner, G. (2009). Bach Flower Remedies for psychological problems and pain: A systematic review. BMC Complementary and Alternative Medicine, 9, 16. https://doi.org/10.1186/1472-6882-9-16

18. The 38 Remedies Quick Reference Guide. (s. f.). The Bach Centre. Recuperado 20 de junio de 2020, de https://www.bachcentre.com/new/en/remedies/the-38remedies/quick-reference-guide/ 